UDC 616-053.36- 092.11-036: 618.177-089.888.11

DOI: 10.21668/health.risk/2017.1.07.eng

\title{
RISK FACTORS AND PREDICTING HEALTH DISORDERS IN INFANTS BORN FROM MONOCYESIS AFTER IN VITRO FERTILIZATION
}

\section{L.A. Pykhtina, O.M. Filkina, N.D. Gadzhimuradova, A.I. Malyshkina, S.B. Nazarov}

Ivanovo Research Institute of Maternity and Childhood named after V.N. Gorodkova, 109 Shuvandina Str., Ivanovo, 153045, Russian Federation

Issues of health state and factors determining it in infants born due to in vitro fertilization (IVF) have been frequently discussed over recent years. The question yet to be answered is whether health disorders are related to burdened pre-morbid state of a mother as per extragenital and obstetric-gynecologic pathology or they are caused by IVF. Selective transfer of only one embryo has tended to increase recently, and, given this trend, specialists all around the world, and in our country as well, face a problem of detecting risk factors which cause health disorders in children born in monocyesis after IVF. Prognostic algorithm for most frequent pathologies is being worked out and it will help to implement targeted and differentiated approach to their prevention. We have completed clinical examination of infants during the first year of their life; all these infants were born in monocyesis after IVF $(n=121)$. We have also questioned and interviewed their parents and analyzed data taken from infants' development records over a year as well. We have applied Wald sequential mathematical analysis to determine risk factors and create prognostic tables comprising most frequent somatic health disorders such as congenital abnormalities, irondeficiency anemia, atopic dermatitis, absence of perinatal CNS damage compensation. We have detected that factors related to a mother's and newborn's health (extragenital morbidity, obstetric-gynecologic case history, pregnancy course) exert their influence on health disorders evolvement in such children during their first year of life; we have also shown that social factors and factors associated with IVF procedure don't have any statistical significance.

Key words: risk factors, health disorders prediction, children from monocyesis, IVF, congenital abnormalities, iron-deficiency anemia, atopic dermatitis, perinatal CNS damages.

There have recently been a lot of discussions on health state and factors determining it in children born due to in-vitro fertilization (IVF) $[1,2,4,6,7,8,17,24,28$, 29]. As per data given by various authors, IVF programs efficiency varies from 20 to $40 \%$, number of born children from this group doesn't exceed $6-25 \%$ of all implanted embryos and $56-78 \%$ of occurred pregnancies $[5,8,25]$. We should point out that conception, fetus development and maturation due to application of auxiliary reproductive technologies takes place under conditions which differ from physiological standards rather substantially $[4,10,21]$. Embryo sensitivity to environment factors at preimplantation stage is considerably high and it can cause fetus pathologies evolvement depending on its gestation stage $[9,10,12,16]$.

As per data taken from the recent

CC Pykhtina L.A., Filkina O.M., Gadzhimuradova N.D., Malyshkina A.I., Nazarov S.B., 2017

Lyudmila A. Pykhtina - Doctor of Medicin, leading researcher at Children Health Protection Department (e-mail: ivniideti@mail.ru; tel.: +7 (493) 233-70-55).

Nadezhda D. Gadzhimuradova - postgraduate at Children Health Protection Department (e-mail: ivniideti@mail.ru; tel.: +7 (493) 233-70-55).

Olga M. Filkina - Doctor of Medicine, professor, Honored Physician of the RF, head of Children Health Protection Department (e-mail: ivniideti@mail.ru; tel.: +7 (493) 233-70-55).

Anna I. Malyshkina - Doctor of Medicine, associate professor, director (e-mail: ivniimid@inbox.ru; tel.: +7 (493) 233-62-63).

Sergey B. Nazarov - Doctor of Medicine, professor, deputy director responsible for research work (e-mail: ivniimid@inbox.ru, tel.: +7 (493) 233-62-63). 
domestic and foreign literature, there are several factors in a mother causing pathological course of neonatal period in children from IVF group. Such factors include burdened obstetric-gynecological case history, late reproductive age, ovary activity stimulation, pre-term delivery, multiple pregnancy, comcomitant somatic pathology, social status, education, and etiology of infertility $[2,5,7,8,11,12,14,15,18,22]$. As per results presented by E.V. Vartanyan [2], risk factors are inflammatory diseases of reproductive system, menstrual function disorders, infertility duration, miscarriages and abortions in case history, surgeries performed on abdominal cavity organs and small pelvis organs, and sexual diseases.

In A.N. Plaksina's opinion [20], health of a child born after IVF pregnancy is determined by the following factors in a mother: missed miscarriage $(20.3 \%)$, miscarriages at various terms $(28.6 \%)$, multiple pregnancy $(11.1 \%)$, and threat of miscarriage $(48.3 \%)$. The author states that women who are older than 30 and have secondary infertility run increased risk for giving birth to a baby with low birth weight and low gestation term.

Some authors say that a method invasiveness is the basic burdening factor which influences infants from IVF group [2, 14]. Other believe that high morbidity and deviations in development of such children are related only to abnormal pregnancy and delivery course $[18,19]$. Influence exerted by different superovulation stimulation schemes in IVF programs on health of a future baby is not sufficiently studied and described in contemporary scientific research [8]. At the same time there are data in literature on higher risks for congenital malformations of genitals in boys whose mothers underwent superovulation stimulation procedure and took progestin during pregnancy [12].

Infertility related to endometriosis is known to be hardly curable, and even IVF application in patients suffering from endometriosis has very low efficiency. Influence exerted by this factor on health of a future baby is not studied sufficiently [10].
Some scientists assume that unfavorable perinatal outcomes are related only to multiple pregnancy after IVF [16]. However some latest research prove that risk of giving birth to a sick infant even in case of monocyesis due to IVF is growing. But we couldn't find enough information on the matter [6, 9, 13, 24, 27].

Several reasons determining reproductive technologies application still exist and later they influence pregnancy course, delivery, and health of a future baby $[4,7,9,15,17,21,25$, 31].

There are practically no scientific works which deal with issues of combined impacts exerted by biological and social factors on somatic health formation in children born due to IVF depending on a number of transferred embryos [13, 22, 26, 27, 30].

Selective transfer of only one embryo has recently tended to increase in many countries, including Russia. And it becomes really essential to study factors causing health disorders risks in order to work out mechanisms of their prediction in children born from monocyesis after IVF. It will allow to apply differentiated and targeted approaches to prevention of most frequent pathologies and reduce risks of their evolvement $[23,30]$.

Our research goal was to highlight risk factors and work out predictive tables for most frequent health disorders (congenital malformations, iron-deficiency anemia, atopic dermatitis, absence of perinatal central nervous system (CNS) damage compensation) in oneyear old children born from monocyesis after in-vitro fertilization.

Data and methods. We examined oneyear old children born from monocyesis after IVF $(n=121)$. Their health was assessed in early neonatal period and after their one-year birthday as per clinical examinaiton results. We assessed morbidity as per appealability to a polyclinic and via dynamic observation over infants including functional methods of examination (according to Order No. 1346n dated December 21, 2012). We collected biological and social case history by copying data from patients' individual cards used at applying to auxiliary reproductive technologies 
(form No. 111-1/u-03); data from a newborn development history (form No. 097/u) and from a child development history (form No. 112/u, form No. 003/u). Certain factors were clarified during questioning and interviewing parents with the use of a specially designed "Questionnaire on detecting social-biological risk factors".

We processed the obtained results statistically using MS Excel XP and Statistica 6.0 software. Discrepancies in relative indices were studied as per Pierson $\chi^{2}$-criterion with Yates correction. Discrepancies were considered authentic at $p<0.05$. We detected correlations between studied indices via calculating Spearmen correlation coefficient $(R)$. We calculated odds relations (OR) and relative risk (RR) for various factors in OpenEpi program with defining $95 \%$ confidence interval (RR, 95\% DI). To detect risk factors for most frequent health disorders and working out a predictive table, we used Wald sequential mathematic analysis [3]. Having proved validity of discrepancy in frequency with which an examined factor occurred in children groups with health disorders and without them $(p<0.05)$, we calculated predictive coefficients (PC) for each factor level. Predictive coefficients were calculated as per formula: $\mathrm{PC}=10 \mathrm{lg}\left(\mathrm{P}_{1} / \mathrm{P}_{2}\right)$ when a factor occurred, $\mathrm{PC}=10 \lg \left(1-\mathrm{P}_{1} / 1-\right.$ $\mathrm{P}_{2}$ ) when a factor was absent, where $\mathrm{P}_{1}$ and $\mathrm{P}_{2}$ was a frequency of a factor occurrence in compared groups. If an obtained value was positive it was an evidence of a factor negative influence.

Results and discussion. We detected that overall morbidity level in children from the basic group during the first year of their life was 1.3. times higher than in the control group (214.9 and 171.1 per 100 people correspondingly), due to higher occurrence frequency of perinatal central nervous system damage (P CNS D) (66.1 and $39.7 \%$ correspondingly; $\left.\chi^{2}=12.97, \quad p=0.000\right)$, congenital malformations, mostly due to minor heart malformations (MHM) (33.1 and $17.4 \%$ correspondingly; $\left.\chi^{2}=7.91, p=0.005\right)$, irondeficiency anemia $(14.0$ and $5.8 \%$ correspondingly; $\chi^{2}=4.13, p=0.042$ ), atopic dermatitis (8.3 and $2.5 \%$ correspondingly; $\chi^{2}=$ 3.16, $p=0.046)$, thymus hyperplasia (7.4 and $1.7 \%$ correspondingly; $\chi^{2}=4.67, p=0.031$ ).

We detected significant factors for evolvement of most frequent health disorders in children basing on the analysis of the following data: biological case history (extragenital morbidity, obstetricgynecological case history, mothers' reproductive function, a child's health state), and social case history (education, social status, occupational hazards of parents, family wealth, and family relations) of children born from monocyesis after IVF, as well as factors related to IVF procedure (IVF protocol duration, a number of IVF attempts, IVF techniques, and embryo quality).

We detected that risk factors causing:

- congenital malformations were: a mother suffering from chronic inflammatory diseases of urinary system (RR 4.4; $95 \%$ CI 2.29-8.37), missed miscarriage in case history (RR 4.4; $95 \%$ CI 2.29-8.37), spontaneous miscarriages in case history (RR 4.0; $95 \% \mathrm{CI}$ 2.03-7.88), male infertility (RR 3.3; $95 \% \mathrm{CI}$ 1.23-8.79), fetoplacental insufficiency (FPI) (RR 3.3; $95 \%$ CI 1.60-6.96), threat of miscarriage (RR 3.2; $95 \%$ CI 1.59-6.62), colpitis during pregnancy (RR 3.2; $95 \%$ CI 1.59-.,26);

- iron-deficiency anemia were: birth after gestation period shorter than 37 weeks (RR 5.1; $95 \%$ CI 2.04-12.60); birth after the third and more pregnancy (RR 4.7; $95 \%$ CI 2.05 10.62); anemia of pregnant (RR 4.3; $95 \%$ CI 1.88-9.93); fetoplacental insufficiency (RR $4.0 ; 95 \%$ CI 1.70-9.41); intraventricular hemorrhages (IVH) (RR 3.2; $95 \%$ CI 1.337.78);

- atopic dermatitis were: burdened allergic case history (RR 8.6; $95 \%$ CI 2.95 25.20), preeclampsia (RR 8.5; $95 \%$ CI 1.709.41), birth after gestation period shorter than 37 weeks (RR 7.0; $95 \%$ CI 1.97-25.44), artificial nutrition since the very birth (RR 3.9; $95 \%$ CI 1.19-12.93);

- absence of perinatal CNS damage compensation by one-year birthday were: birth after gestation period shorter than 37 weeks 
(RR 3.3; $95 \%$ CI 1.90-5.84), fetus growth retardation ( $\mathrm{RR} 3.1 ; 95 \%$ CI 1.94-5.17), gestational arterial hypertension (RR 3.1; $95 \%$ CI 1.95-5.16), chronic adnexa inflammation in case history (RR 2.8; $95 \%$ CI 1.46-5.34), neurocirculatory dystonia (NCD) in a mother (RR 1.9; $95 \%$ CI 1.40-2.70), fetus growth retardation (RR 1.9; $95 \%$ CI 1.34-2.66), intraventricular hemorrhages of the 2nd degree in neonatal period (RR 2.7; $95 \%$ CI 1.574.60), iron-deficiency anemia in a child (RR 1.9 ; $95 \%$ CI 1.09-3.53).

We detected that the greatest influence on evolvement of most frequent health disorders in children born from monocyesis after IVF was exerted by biological factors related to health of a mother and a newborn. Social factors had no statistic significance.
We analyzed factors influencing evolvement of most frequent health disorders in examined children related to IVF procedure (table 1).

We detected that factors determined by the very procedure had no statistically significant influence on evolvement of most frequent health disorders in children born from monocyesis after IVF.

Basing on the highlighted biological risk factors for children born from monocyesis after IVF, we worked out formalized tables for predicting congenital malformations evolvement, iron-deficiency anemia, atopic dermatitis, absence of perinatal central nervous system damage compensation by one-year birthday (table 2).

Factors, related to IVF procedure, for most frequent health disorders in

Table 1 children born from monocyesis, abs. (\%)

\begin{tabular}{|c|c|c|c|c|c|c|c|c|}
\hline \multirow{3}{*}{ Index } & \multicolumn{8}{|c|}{ Factor } \\
\hline & \multicolumn{2}{|c|}{$\begin{array}{l}\text { Congenital mal- } \\
\text { formations }\end{array}$} & \multicolumn{2}{|c|}{$\begin{array}{c}\text { Congenital mal- } \\
\text { formations }\end{array}$} & \multicolumn{2}{|c|}{$\begin{array}{c}\text { Congenital mal- } \\
\text { formations }\end{array}$} & \multicolumn{2}{|c|}{$\begin{array}{c}\text { Congenital malfor- } \\
\text { mations }\end{array}$} \\
\hline & yes & no & yes & no & yes & no & yes & no \\
\hline & $n=21$ & $n=84$ & $n=16$ & $n=89$ & $n=10$ & $n=95$ & $n=12$ & $n=93$ \\
\hline \multicolumn{9}{|l|}{ IVF protocol: } \\
\hline short & $\begin{array}{c}2 \\
(9,5)\end{array}$ & $\begin{array}{c}13 \\
(15,5)\end{array}$ & $\begin{array}{c}4 \\
(25,0)\end{array}$ & $\begin{array}{c}11 \\
(12,4)\end{array}$ & $\begin{array}{c}1 \\
(10,0)\end{array}$ & $\begin{array}{c}14 \\
(14,7)\end{array}$ & $\begin{array}{c}1 \\
(8,3)\end{array}$ & $\begin{array}{c}14 \\
(15,1)\end{array}$ \\
\hline long & $\begin{array}{c}19 \\
(90,5)\end{array}$ & $\begin{array}{c}71 \\
(84,5)\end{array}$ & $\begin{array}{c}12 \\
(75,0)\end{array}$ & $\begin{array}{c}78 \\
(87,6)\end{array}$ & $\begin{array}{c}9 \\
(90,0)\end{array}$ & $\begin{array}{c}81 \\
(85,3)\end{array}$ & $\begin{array}{c}11 \\
(91,7)\end{array}$ & $\begin{array}{c}79 \\
(84,9)\end{array}$ \\
\hline \multicolumn{9}{|c|}{ Number of IVF attempts: } \\
\hline $1 \mathrm{st}$ & $\begin{array}{c}14 \\
(66,7)\end{array}$ & $\begin{array}{c}61 \\
(72,6)\end{array}$ & $\begin{array}{c}11 \\
(68,8)\end{array}$ & $\begin{array}{c}64 \\
(71,9)\end{array}$ & $\begin{array}{c}7 \\
(70,0)\end{array}$ & $\begin{array}{c}68 \\
(71,6)\end{array}$ & $\begin{array}{c}6 \\
(50,0)\end{array}$ & $\begin{array}{c}61 \\
(65,6)\end{array}$ \\
\hline More than 2 & $\begin{array}{c}7 \\
(33,3)\end{array}$ & $\begin{array}{c}23 \\
(27,4)\end{array}$ & $\begin{array}{c}5 \\
(31,3)\end{array}$ & $\begin{array}{c}33 \\
(37,1)\end{array}$ & $\begin{array}{c}3 \\
(30,0)\end{array}$ & $\begin{array}{c}35 \\
(36,8)\end{array}$ & $\begin{array}{c}6 \\
(50,0)\end{array}$ & $\begin{array}{c}32 \\
(34,4)\end{array}$ \\
\hline \multicolumn{9}{|l|}{ IVF techniques: } \\
\hline IVF & $\begin{array}{c}8 \\
(38,1)\end{array}$ & $\begin{array}{c}31 \\
(36,9)\end{array}$ & $\begin{array}{c}8 \\
(50,0)\end{array}$ & $\begin{array}{c}31 \\
(34,8)\end{array}$ & $\begin{array}{c}2 \\
(20,0)\end{array}$ & $\begin{array}{c}37 \\
(38,9)\end{array}$ & $\begin{array}{c}3 \\
(25,0)\end{array}$ & $\begin{array}{c}36 \\
(38,7)\end{array}$ \\
\hline IVF + ICSI & $\begin{array}{c}13 \\
(61,9)\end{array}$ & $\begin{array}{c}53 \\
(63,1)\end{array}$ & $\begin{array}{c}8 \\
(50,0)\end{array}$ & $\begin{array}{c}58 \\
(65,2)\end{array}$ & $\begin{array}{c}8 \\
(80,0)\end{array}$ & $\begin{array}{c}58 \\
(61,1)\end{array}$ & $\begin{array}{c}9 \\
(75,0)\end{array}$ & $\begin{array}{c}57 \\
(61,3)\end{array}$ \\
\hline \multicolumn{9}{|l|}{ Quality of embryos: } \\
\hline excellent, A type & $\begin{array}{c}15 \\
(71,4)\end{array}$ & $\begin{array}{c}63 \\
(75,0)\end{array}$ & $\begin{array}{c}10 \\
(62,5)\end{array}$ & $\begin{array}{c}68 \\
(76,4)\end{array}$ & $\begin{array}{c}5 \\
(50,0)\end{array}$ & $\begin{array}{c}73 \\
(76,8)\end{array}$ & $\begin{array}{c}6 \\
(50,0)\end{array}$ & $\begin{array}{c}72 \\
(77,4)\end{array}$ \\
\hline good, B type & $\begin{array}{c}6 \\
(28,6)\end{array}$ & $\begin{array}{c}19 \\
(25,0)\end{array}$ & $\begin{array}{c}6 \\
(37,5)\end{array}$ & $\begin{array}{c}19 \\
(23,6)\end{array}$ & $\begin{array}{c}5 \\
(50,0)\end{array}$ & $\begin{array}{c}20 \\
(23,2)\end{array}$ & $\begin{array}{c}6 \\
(50,0)\end{array}$ & $\begin{array}{c}21 \\
(22,6)\end{array}$ \\
\hline
\end{tabular}


Table 2

Predictive table for health disorders in children born from monocyesis after IVF during the first year of their life

\begin{tabular}{|c|c|c|c|c|}
\hline \multirow[b]{2}{*}{ Risk factors } & \multicolumn{4}{|c|}{ Predictive coefficients (PC) } \\
\hline & $\begin{array}{l}\text { Congenital } \\
\text { malfor- } \\
\text { mations }\end{array}$ & $\begin{array}{l}\text { Congenital } \\
\text { malformations }\end{array}$ & $\begin{array}{l}\text { Congenital } \\
\text { malfor- } \\
\text { mations }\end{array}$ & $\begin{array}{c}\text { Congenital } \\
\text { malformations }\end{array}$ \\
\hline 1 & 2 & 3 & 4 & 5 \\
\hline \multicolumn{5}{|c|}{ Extragenital pathology in a mother } \\
\hline \multicolumn{5}{|c|}{ Chronic inflammatory diseases of urinary system: } \\
\hline yes & $+7,00$ & - & - & - \\
\hline no & $-0,75$ & - & - & - \\
\hline \multicolumn{5}{|l|}{ Burdened allergic case history: } \\
\hline yes & - & - & $+4,93$ & - \\
\hline no & - & - & $-2,61$ & - \\
\hline \multicolumn{5}{|c|}{ NCD (VNS somatoform disorder): } \\
\hline yes & - & - & - & $+7,24$ \\
\hline no & - & - & - & $-0,78$ \\
\hline \multicolumn{5}{|c|}{ Obstetric-gynecological case history } \\
\hline \multirow{2}{*}{\multicolumn{5}{|c|}{\begin{tabular}{l|l|l} 
Chronic adnexa inflammation in case history: & \multicolumn{2}{|c}{} \\
\cline { 2 - 3 } yes & - & $\ldots$
\end{tabular}}} \\
\hline & - & - & - & $+2,83$ \\
\hline no & - & - & - & $-2,13$ \\
\hline \multicolumn{5}{|l|}{ Endometriosis in case history: } \\
\hline yes & $+2,98$ & - & - & - \\
\hline no & $-0,99$ & - & - & - \\
\hline \multicolumn{5}{|c|}{ Spontaneous miscarriages in case history: } \\
\hline yes & $+5,09$ & - & - & - \\
\hline no & $-1,23$ & - & - & - \\
\hline \multicolumn{5}{|c|}{$\begin{array}{l}\text { Missed miscarriage in case history: } \\
\text { ves }\end{array}$} \\
\hline yes & $+7,00$ & - & - & - \\
\hline no & $-0,75$ & - & - & - \\
\hline \multicolumn{5}{|l|}{ Male infertility: } \\
\hline yes & $+3,01$ & - & - & - \\
\hline no & $-1,63$ & - & - & - \\
\hline \multicolumn{5}{|c|}{ This pregnancy course } \\
\hline \multirow{2}{*}{$\begin{array}{l}\text { Fetoplacental insufficiency: } \\
\text { yes }\end{array}$} & & & & \\
\hline & $+4,89$ & $+3,74$ & - & - \\
\hline no & $-0,68$ & $-1,63$ & - & - \\
\hline \multicolumn{5}{|l|}{ Fetus growth retardation: } \\
\hline yes & - & - & - & $+5,08$ \\
\hline no & - & - & - & $-0,54$ \\
\hline \multicolumn{5}{|l|}{ Preeclampsia: } \\
\hline yes & - & - & $+5,12$ & - \\
\hline no & - & - & $-1,70$ & - \\
\hline \multicolumn{5}{|l|}{ Threat of miscarriage: } \\
\hline yes & $+4,21$ & - & - & - \\
\hline no & $-0,97$ & - & - & - \\
\hline \multicolumn{5}{|l|}{ Gestational arterial hypertension: } \\
\hline yes & - & - & - & $+6,09$ \\
\hline no & - & - & - & $-0,39$ \\
\hline \multicolumn{5}{|l|}{ Anemia of pregnant: } \\
\hline yes & - & $+5,22$ & - & - \\
\hline no & - & $-0,96$ & - & - \\
\hline \multicolumn{5}{|l|}{ Colpitis during pregnancy: } \\
\hline yes & $+3,36$ & - & - & - \\
\hline no & $-1,01$ & - & - & - \\
\hline
\end{tabular}


Risk factors and predicting health disorders in infants born from monocyesis after in vitro fertilization

Continuation of table 2

\begin{tabular}{|c|c|c|c|c|}
\hline \multicolumn{5}{|c|}{ A child's health state } \\
\hline \multirow{2}{*}{$\begin{array}{l}\text { Birth after gestation period shorted than } 34 \text { weeks: } \\
\text { yes }\end{array}$} & & & & \\
\hline & $+4,22$ & - & - & - \\
\hline \multirow{3}{*}{$\begin{array}{l}\text { no } \\
\text { Birth after gestation period shorted than } 37 \text { weeks: } \\
\text { yes }\end{array}$} & $-2,44$ & 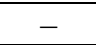 & - & - \\
\hline & & & & \\
\hline & - & $+3,79$ & $+3,93$ & $+2,66$ \\
\hline no & - & $-2,38$ & $-3,52$ & $-0,86$ \\
\hline \multirow{2}{*}{$\begin{array}{l}\text { Birth after the 3rd or more pregnancy: } \\
\text { yes }\end{array}$} & & & & \\
\hline & - & $+5,82$ & - & - \\
\hline no & 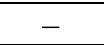 & $-2,39$ & - & 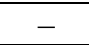 \\
\hline \multirow{2}{*}{$\begin{array}{l}\text { Artificial feeding since the very birth: } \\
\text { yes }\end{array}$} & & & & \\
\hline & - & - & $+4,55$ & - \\
\hline no & - & - & $-5,68$ & - \\
\hline \multirow{2}{*}{$\begin{array}{l}\text { Non-traumatic intraventricular hemorrhages of the } \\
\text { 2nd degree in case history: } \\
\text { yes }\end{array}$} & & & & \\
\hline & - & $+7,60$ & - & $+6,09$ \\
\hline no & - & $-1,99$ & - & $-0,39$ \\
\hline \multirow{2}{*}{$\begin{array}{l}\text { Iron-deficiency anemia: } \\
\text { yes }\end{array}$} & & & & \\
\hline & - & - & - & $+6,88$ \\
\hline no & - & - & - & $-0,53$ \\
\hline
\end{tabular}

An individual forecast is determined by a sum of predictive coefficients (PC). We used Wald's formula to determine predictive threshold (PT) value which allows to assess validity of health disorders evolvement in children born from monocyesis after IVF during the first year of their life (congenital malformations, iron-deficiency anemia, atopic dermatitis, perinatal central nervous system damage compensation) [17]. We considered not more than a $5 \%$ probability of an error in a forecast to be acceptable and determined that predictive threshold (PT) for a possibility of these health disorders evolvement was equal to +13 , and their absence, -13 .

If the predictive coefficients sum is equal or more than +13 , than the forecast is unfavorable, and one can predict evolvement of congenital malformations (as per PC sum in the second column); iron-deficiency anemia during the first year of their life (as per PC sun in the third column); atopic dermatitis (as per PC sum in the fourth column); absence of perinatal CNS damage compensation by oneyear birthday (as per PC sum in the fifth column).

If the PC sum is equal or less than -13 , the forecast is favorable, and one can predict absence of such health disorder evolvement.
If the $\mathrm{PC}$ sum is within +12 to -12 range, than the forecast is uncertain, and we don't have enough data to make a decision on it (attention group).

We recommend pediatricians to include children with unfavorable forecasts into a risk group as per such health disorders evolvement and assign preventive activities which can lower such risks evolvement.

Conclusions. So, in the course of our research we detected biological risk factors for most frequent disorders in somatic health of children born from monocyesis after IVF during the first year of their life; such disorders include congenital malformations, iron-deficiency anemia, atopic dermatitis, consequences of perinatal CNS damage. We detected that somatic health formation in children born from monocyesis after IVF during the first year of their life is greatly influenced by factors related to a mother's health (extragenital morbidity, obstetricgynecological case history, pregnancy course). and a newborn's health. Here social factors and factors determined by IVF procedure itself don't exert any statistically significant influence.

We worked out predictive tables which can help to predict evolvement of health disorders in a child born from monocyesis 
after IVF just after its birth; we hope these neonatologists, district pediatricians, and tables will be suitable for practical use by family doctors.

\section{References}

1. Baranov A.A. Spravka o sostoyanii zdorov'ya detei, rodivshikhsya v rezul'tate ispol'zovaniya vspomogatel'nykh reproduktivnykh tekhnologii, v tom chisle EKO [Information on health state of children born due to assisted reproductive technologies including IVF]. Soyuz pediatrov Rossii. Available at: http: //www.pediatr-russia.ru/node/124 (02.10.2016) (in Russian).

2. Vartanyan E.V. Vozmozhnosti preodoleniya povtornykh neudach $\mathrm{v}$ protsedurakh vspomogatel'nykh reproduktivnykh tekhnologii [Overcoming the Possibility of Repeated IVF Failure]. Vestnik Ural'skoi meditsinskoi akademicheskoi nauki, 2013, vol. 45, no. 3, pp. 56-62 (in Russian).

3. Gubler E.V. Vychislitel'nye metody analiza i raspoznavaniya patologicheskikh protsessov [Computational techniques of pathologic processes analysis and recognition]. Leningrad, Meditsina Publ., 1978, 94 p. (in Russian).

4. Zhirnov V.A., Dmitrieva M.V., Rustyanova D.R. Analiz somaticheskogo statusa novorozhdennykh detei, zachatykh posredstvom ekstrakorporal'nogo oplodotvoreniya [Analyzing somatic state of newborns, conceived via IVF]. Molodoi uchenyi: vyzovy i perspektivy: materialy VI Mezhdunarodnoi nauchno-prakticheskoi konferentsii [Young scientist: challenges and prospects: materials of VI International theoretical and practical conference]. In: N.R. Krasovskaya ed. Moscow, 2016, pp. 67-71 (in Russian).

5. Zdanovskii V.M., Vityazeva I.I. Techenie i iskhod beremennostei posle lecheniya besplodiya metodami vspomogatel'noi reproduktsii (MVR) [Pregnancy course and outcome after treating infertility with assisted reproductive technologies]. Problemy reproduktsii, 2000, no. 3, pp. 55-56 (in Russian).

6. Gadzhimuradova N.D., Pykhtina L.A., Fil'kina O.M., Malyshkina A.I. Zdorov'e detei, rozhdennykh posle primeneniya ekstrakorporal'nogo oplodotvoreniya, i ikh materei [The health of children born after extracorporeal fertilization and their mother`s health]. Vrach-aspirant, 2015, vol. 71, no 4, pp. 5156 (in Russian).

7. Evert L.S., Galonskii V.G., Tepper E.A., Volynkina A.I., Tarasova N.V. Iskhody beremennosti i sostoyanie zdorov'ya detei, rozhdennykh posle primeneniya vspomogatel'nykh reproduktivnykh tekhnologii [Pregnancy outcomes and health of children born following assisted reproductive technologies] Sibirskii meditsinskii zhurnal (g. Tomsk), 2013, vol. 28, no. 1, pp. 65-69 (in Russian).

8. Kalinina E.A., Alieva K.U., Strel'chenko M.B. Nauchno-klinicheskii analiz raboty otdeleniya vspomogatel'nykh tekhnologii v lechenii besplodiya v 2011 godu [Clinico-scientific analysis summarizing the work of the department for assisted reproductive technology for the year 2011]. Akusherstvo $i$ ginekologiya, 2013, no. 1, pp. 81-84 (in Russian).

9. Kinsht D.A., Soboleva M.K., Aizikovich I.V. Zdorov'e novorozhdennykh ot odnoplodnoi indutsirovannoi beremennosti: sobstvennyi opyt nablyudeniya MTs «Avitsenna» [The health of infants from singleton induced pregnancy: own experience of observing medical center «Avicenna»]. Reproduktivnaya meditsina, 2014, vol. 20, no. 3-4, pp. 18-23 (in Russian).

10. Korsak V.S., Vasil'eva O.V., Isakova E.V. Endometrioz i VRT (obzor literatury) [Endometriosis and ART (literature overview)]. Problemy reproduktsii, 2006, no. 3, pp. 41-46 (in Russian).

11. Korsak V.S., Smirnova A.A., Shurygina O.V. Registr tsentrov VRT v Rossii. Otchet za 2011 god [ART centers register in Russia. 2011 report]. Problemy reproduktsii, 2013, no. 5, pp. 7-16 (in Russian).

12. Krasnopol'skaya K.V., Kabanova D.I., Kalugina A.S. Effektivnost' ekstrakorporal'nogo oplodotvoreniya u patsientok s sindromom polikistoznykh yaichnikov i yaichnikovoi giperandrogeniei [IVF efficiency in patients suffering from polycystic ovary syndrome and ovarian hyperandrogenism]. Akusherstvo i ginekologiya, 2003, no. 1, pp. 57-61 (in Russian).

13. Krasnoshchoka O.E., Smol'nikova V.Yu., Kalinina E.A. Klinicheskie i embriologicheskie aspekty selektivnogo perenosa odnogo embriona [Clinical and embryological aspects of elective single embryo 
transfer]. Problemy reproduktsii, 2015, vol. 21, no. 2, pp. 51-57 (in Russian).

14. Krstich E.V., Krasnopol'skaya K.V., Kabanova D.I. Novye podkhody k povysheniyu effektivnosti EKO u zhenshchin starshego reproduktivnogo vozrasta [New approaches to enhancing THE EFFICIENCY OF in vitro fertilization in old reproductive age women]. Akusherstvo i ginekologiya, 2010, no. 2, pp. 48-53 (in Russian).

15. Lysenko A.V., Markelova M.I., Sudakova N.M. Analiz faktorov riska beremennosti i rannego neonatal'nogo perioda novorozhdennykh posle vspomogatel'nykh reproduk $\neg$ tivnykh tekhnologii [Analyzing risk factors for pregnancy and early neonatal period of newborns after assisted reproductive technologies]. Sovremennye nauchnye issledovaniya i innovatsii, 2013, no. 1. Available at: http: //web.snauka.ru/issues/2013/01/19773 (10.10.2016) (in Russian).

16. Maslyanyuk N.A. Sostoyanie novorozhdennykh detei i ikh dal'neishee razvitie pri mnogoplodnoi beremennosti posle ekstrakorporal'nogo oplodotvoreniya: avtoref. dis. ... kand. med. nauk [Newborns state and their further development at multiple pregnancy after IVF: abstract of a thesis ... candidate of medical sciences]. St. Petersburg, 2005, 24 p. (in Russian).

17. Mikheeva E.M., Penkina N.I. Zdorov'e detei, rozhdennykh s ispol'zovaniem vspomogatel'nykh reproduktivnykh tekhnologii [The health status of children conceived with assisted reproductive technologies]. Prakticheskaya meditsina, 2014, vol. 85, no. 9, pp. 47-51 (in Russian).

18. Vyalikova Yu.V., Alieva A.Ya., Naiko Yu.V., Shintaev T.K. Osobennosti beremennosti i rodov u patsientok posle ekstrakorporal'nogo oplodotvoreniya [Peculiarities of pregnancy and labor in patients after IVF]. Byulleten' meditsinskikh internet-konferentsii, 2015, vol. 5, no. 5, pp. 442 (in Russian).

19. Shchetinina N.S., Kuz'michev L.N., Burlev V.A., Onishchenko A.S., Il'yasova N.A. Otsenka effektivnosti programmy EKO: den' perenosa embrionov v polost' matki i pokazateli kontroliruemoi induktsii ovulyatsii [Assessment of IVF/ICSI efficasy: the day of embryo transfer and parameters of controlled ovarian hyperstimulation]. Problemy reproduktsii, 2011, no. 3, pp. 56-61 (in Russian).

20. Plaksina A.N. Prognozirovanie zdorov'ya i kachestva zhizni detei, rozhdennykh s pomoshch'yu vspomogatel'nykh reproduktivnykh tekhnologii: dis. ... kand. med. Nauk [Predicting health and life quality for children, born due to assisted reproductive technologies: abstract of a thesis ... candidate of medical sciences]. Ekaterinburg, 2011, 174 p. (in Russian).

21. Saidova R.A., Guseinova Z.S. Znachenie predgravidarnoi podgotovki v profilaktike sindroma poteri ploda u bol'nykh s giperandrogeniei [Pregravid preparation in prophylaxis of syndrome of fetus loss in patients with hyperandrogenism]. Problemy zhenskogo zdorov'ya, 2011, vol. 6, no. 1, pp. 25-30 (in Russian).

22. Rudakova E.B., Loboda O.A., Poltoraka E.V., Burova O.M., Pilipenko M.A. Faktory riska neudach i embrionicheskikh poter' pri ekstrakorporal'nom oplodotvorenii [Risk factors of failures and embryonic losses under in vitro fertilization]. Sibirskii meditsinskii zhurnal (g. Tomsk), 2008, vol. 23, no. 41, pp. 14-17 (in Russian).

23. Fil'kina O.M., Pykhtina L.A., Vorob'eva E.A., Kocherova O.Yu., Dolotova N.V., Shanina T.G. Faktory riska otklonenii fizicheskogo razvitiya u detei rannego vozrasta s perinatal'nymi porazheniyami tsentral'noi nervnoi sistemy [The risk factors of dapartures in physical development in children of early age with perinatal affection of central nervous system]. Lechenie i profilaktika, 2015, vol. 13, no. 1, pp. 16-21 (in Russian).

24. Källén B., Finnström O., Lindam A., Nilsson E., Nygren Karl-Gösta, Olausson P.O. Cancer risk in children and young adults conceived by in vitro fertilization. Pediatrics, 2010, vol. 126, pp. 270-276.

25. Elective single embryo transfer. Fertil Steril, 2012, vol. 97, no. 4, pp. 835-842. Available at: https: //www.asrm.org/Elective Single Embryo Transfer/ (15.10.2016)

26. Gelbaya T.A., Tsoumpou I., Nardo L.G. The likelihood of live birth and multiple birth after single versus double embryo transfer at the cleavage stage: a systematic review and meta-analysis. Fertility and Sterility, 2010, vol. 94, no. 3, pp. 936-945.

27. Pandey S., Shetty A., Hamilton M., Bhattacharya S., Maheshwari A. Obstetric and perinatal outcomes in singleton pregnnancies resulting from IVF/ ICSI: a systematic review and metaanalysis. Hum. Reprod. Update, 2012, vol. 18, no. 5, pp. 485-503. DOI: 10.1093/humupd/dms018.

28. Zhu J.L., Hvidtjørn D., Basso O., Obel C., Thorsen P., Uldall P., Olsen J. Parental infertility and 
cerebralpalsy in children. Human Reproduction, 2010, vol. 25, no. 12, pp. 3142-3145. DOI: 10.1093/humrep/deq206

29. Gosden R., Trasler J., Lucifero D., Faddy M. Rare congenital disorders, imprinted genes, and assisted reproductive technology. Lancet, 2003, vol. 361, pp. 1975-1977.

30. Tararbit K., Lelong N., Thieulin A.-C., Houyel L., Bonnet D., Goffinet F., Khoshnood B. The risk for four specific congenital heart defects associated with assisted reproductive techniques: a population-based evaluation. Human Reproduction, 2013, vol. 28, pp. 367-374.

31. World Population Prospects: The 2015 Revision: Key Findings and Advans Tables. New York, United Nations, 2015. Available at: //esa.un.org/unpd/wpp/publications/files/key_findings_wpp_2015.pdf (18.10.2016).

Pykhtina L.A., Filkina O.M., Gadzhimuradova N.D., Malyshkina A.I., Nazarov S.B. Risk factors and predicting health disorders in infants born from monocyesis after in vitro fertilization. Health Risk Analysis, 2017, no. 1, pp. 52-60. DOI: 10.21668/health.risk/2017.1.07.eng

Received: 13.01.2017

Accepted: 10.03 .2017

Published: 30.03.2017 